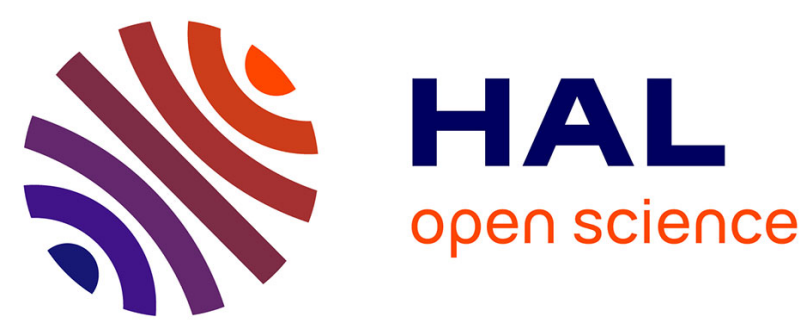

\title{
Evaluation of reticulated gelatin-hibiscus-propolis against intestinal commensal species commonly associated with urinary tract infections
}

Maïwenn Olier, Soraya Sekkal, Cherryl Harkat, Hélène Eutamène, Vassilia Theodorou

\section{To cite this version:}

Maïwenn Olier, Soraya Sekkal, Cherryl Harkat, Hélène Eutamène, Vassilia Theodorou. Evaluation of reticulated gelatin-hibiscus-propolis against intestinal commensal species commonly associated with urinary tract infections. Future Microbiology, 2017, 12 (6), pp.505-513. 10.2217/fmb-2016-0175 . hal-01602765

\section{HAL Id: hal-01602765 https://hal.science/hal-01602765}

Submitted on 3 Jun 2021

HAL is a multi-disciplinary open access archive for the deposit and dissemination of scientific research documents, whether they are published or not. The documents may come from teaching and research institutions in France or abroad, or from public or private research centers.
L'archive ouverte pluridisciplinaire HAL, est destinée au dépôt et à la diffusion de documents scientifiques de niveau recherche, publiés ou non, émanant des établissements d'enseignement et de recherche français ou étrangers, des laboratoires publics ou privés. 


\section{Evaluation of reticulated gelatin-hibiscus- propolis against intestinal commensal species commonly associated with urinary tract infections}

\author{
Maïwenn Olier*,1, Soraya Sekkal', Cherryl Harkat ${ }^{1}$, Hélène Eutamene ${ }^{1}$ \\ \& Vassilia Theodorou ${ }^{* * 1}$
}

\begin{abstract}
Aim: Reticulated gelatin (RG), hibiscus and propolis (RGHP) is a medical device that can reduce the bacterial adherence to epithelial cultured cells and invasion by enteropathogens, thus gathering relevant properties to decrease the risk of urinary tract infections (UTIs). We aimed at evaluating in Wistar rats the efficacy of RGHP, RG and vehicle against intestinal commensals commonly involved in UTIs. Methods: Animals received orally (with supplemental $\mathrm{Na}_{2} \mathrm{CO}_{3}$ ): RGHP $1540 \mathrm{mg} /$ day/rat; RG $500 \mathrm{mg} /$ day/rat or vehicle. Results: RGHP significantly reduced fecal Escherichia coli and Enterococcus spp. levels without affecting other targeted Enterobacteriaceae. The antagonistic property of RGHP was confirmed in streptomycin-pretreated rats highly colonized with a human commensal $E$. coli strain with uropathogenic potential. Conclusion: RGHP may decrease the risk of UTIs by reducing colonization by opportunistic uropathogens.
\end{abstract}

First draft submitted: 31 August 2016; Accepted for publication: 24 January 2017; Published online: 3 March 2017

Urinary tract infections (UTIs) are among the most common bacterial infections in humans and they primarily affect women. Indeed, it has been reported that $40-60 \%$ of adult women will experience at least one uncomplicated UTI episode during their lifetime [1]. In total, approximately 150 million people worldwide are diagnosed with UTIs every year, and this represents more than 10 million cases in Western Europe alone [2,3].

The most common causative pathogen of UTIs is Escherichia coli, which is associated with more than $65 \%$ of all cases [3]. E. coli causing UTIs are referred to as uropathogenic E. coli (UPEC) and extraintestinal pathogenic E. coli. Other uropathogens implicated in uncomplicated UTIs include Klebsiella pneumoniae, Staphylococcus saprophyticus, Enterococcus faecalis and Proteus mirabilis. Following E. coli, Enterococcus spp. are the second most prevalent causative pathogens in complicated UTIs which are usually associated with factors such as urinary tract abnormalities, indwelling catheters, reduced host defense mechanisms (decreased immune function in patients such as the frail and/or the elderly, pregnant women and patients who are immunosuppressed or immunocompromised) or as a consequence of previous exposure to antibiotics leading to bacterial resistance [3]. The intestinal microbiota is the most common immediate source of uropathogens [4-7]. In the case of recurrent UTIs, the UPEC strain responsible for the UTI has been shown to be present in the intestinal microbiota of $78 \%$ of women [7].

Despite considerable research efforts in this disease setting and particularly in identification of traits that differentiate uropathogens from commensals, the mechanisms that govern the transition

'Neuro-Gastroenterology \& Nutrition, UMR 1331 Toxalim, INRA/INPT/UPS, 180 Chemin de Tournefeuille, 31027 Toulouse Cedex 3,

France

*Author for correspondence: Tel.: +33 582066 457; Fax: +33 561285 145; maiwenn.olier@inra.fr

**Author for correspondence: vassilia.theodorou@inra.fr

\section{KEYWORDS}

- commensal Escherichia coli

- Enterococcus

- fecal colonization • fecal opportunistic uropathogens

- reticulated gelatin

- urinary tract infections

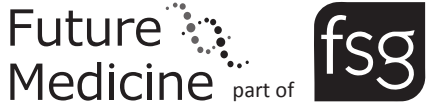


from commensalism to pathogenicity of potentially uropathogenic strains are not well understood. With regard to E. coli, its structure is clonal and the strains are distributed among five main phylogroups (A, B1, B2, D and E) [8,9]. Strains of the various phylogroups differ in their phenotypic characteristics, in their ecological niches as well as in their propensity to cause disease [10]. Uropathogenic isolates are predominantly from the phylogenetic group B2 and take advantage of host behavior and susceptibility by employing virulence factors or fitness factors that facilitate bacterial growth, biofilm formation and persistence in the urinary tract [3,11-13]. These factors include mainly adhesins (e.g., type 1 fimbriae), immune-evasion-related determinants (e.g., polysaccharide capsules K1 and K5), iron- and zinc-acquisition systems (e.g., siderophores, ZupT), biofilm formation components and toxins (e.g., endotoxin, hemolysin) [3,13]. Factors with similar functions are found among other uropathogens [3,14].

Reticulated gelatin hibiscus and propolis (RGHP) is an oral class III medical device recently approved in the EU to control and prevent UTIs [15]. RGHP contains a combination of natural products: reticulated gelatin (RG), Hibiscus sabdariffa and propolis. The RG forms a protective biofilm on the intestinal wall and is commonly used to control and reduce the symptoms associated with acute diarrhea by reducing the adhesion and proliferation of bacterial pathogens in the mucosa $[16,17]$. It has also been reported that $R G$ reduces the immune response of Caco-2 cells stimulated with Gram-negative bacterial lipopolysaccharide [18] and decreases the severity of colitis in mice exposed to dextran sodium sulfate [19]. In this chemically induced colitis model, treatment with RG preserved the appearance and elasticity of the intestinal mucus layer, and it partially reduced colitisinduced overgrowth of Enterobacteriaceae and Enterococcus spp. [19]. In vitro, RG protected intestinal epithelial cells against the adhesion and invasion of the pathogens Salmonella typhimurium and E. coli (ATCC 8739), probably through physical protection of the intestinal epithelium [20].

Considering that UTIs may result from extra-intestinal colonization of opportunistic gut commensals [7], and that the antimicrobial properties attributed to the basal components of RGHP are essentially based on in vitro studies, the first aim of this study was to evaluate the protective properties of RGHP in vivo against fecal species whose strains are known to be involved in the etiology of UTIs. The focus of the evaluation is the key bacteria $E$. coli, a species within which opportunist uropathogens have currently acquired resistance mechanisms against $\beta$-lactams and fluoroquinolones [21,22] . The overall objective of the study was also to provide further insight into a potential new nonantibiotic-based strategy able to limit the source of fecal contamination leading to UTIs.

\section{Material \& methods \\ - Compound}

RGHP was kindly provided by Novintethical Pharma SAGL (Lugano, Switzerland).

\section{- Bacterial strain}

E. coli $\mathrm{M} 2 / 29 \mathrm{~S} \mathrm{tr}^{\mathrm{R}}$ strain isolated from the feces of a healthy human was kindly provided by Ulrich Dobrindt (Münster, Germany). The mutation on the ribosomal subunit protein $\mathrm{S} 12$ (K42R) conferred the streptomycin-resistance phenotype $\left(\mathrm{Str}^{\mathrm{R}}\right)$ used in vivo to facilitate gut colonization of the streptomycin-treated rats as previously described [23]. This commensal E. coli strain shares some common phylogenic characteristics of the archetypal UPEC strains: $\mathrm{B} 2$ phylogenetic group, serotype (O1, K1), genes encoding adhesins such as fimH (subunit of adhesin type 1 fimbriae) sfalfoc (S fimbriae, F1C fimbriae) and $p a p C$ (subunit of adhesin P-pili) and iron-uptake-related genes (aer). The E. coli $\mathrm{M} 2 / 29 \mathrm{Str}^{\mathrm{R}}$ strain inoculated to mice $\left(10^{8} \mathrm{CFU}\right.$ subcutaneously according to the mouse model developed by Picard et al. [11]) was able to colonize urine, blood (heart), kidney and spleen $6 \mathrm{~h}$ after the inoculation.

\section{- Animals}

Female Wistar rats (Janvier S.A., Le Genest St. Isle, France) of 175-200 g were housed at the Animal Care Facility of Toxalim (Experimental Zootechnique Platform) with free access to food and water throughout the study. All animal experiments were conducted in accordance with the guidelines laid out in the 'Guide for the Care and Use of Laboratory Animals' [24] and complied with EU regulations. The Toxalim animal facility, named EZOP (INRA, UMR 1331, Toulouse), is licensed by the French Ministry of Agriculture (agreement no. B31.555.13). All animal experiments were also conducted with approval from the Regional 
Animal Care Committee (CNREEA no. 1; $\mathrm{MP} / 03 / 62 / 11 / 11)$.

\section{- Experimental design}

The impact of RGHP treatment on intestinal bacterial species commonly associated with UTIs was investigated in two experiments. The first involved female rats with intestinal microbiota not altered by streptomycin (Figure 1A). After a 7-day period of acclimatization into animal facility in order to stabilize rat intestinal microbiota, the control group (vehicle, $\mathrm{n}=6$ ) was treated with water supplemented with $\mathrm{Na}_{2} \mathrm{CO}_{3}$ $(5 \%)$ and the treated groups $(n=6)$ with RGHP or RG twice daily, from day 7 to day 10 , in other words, usual adult human posology adapted to the rat metabolism. The second involved female rats highly colonized with $\mathrm{M} 2 / 29 \mathrm{Str}^{\mathrm{R}}$ E. coli strain (Figure 1B); the same design was applied with three groups of ten rats except that drinking water was supplemented with streptomycin sulfate ( $5 \mathrm{~g} / \mathrm{l}$, Sigma-Aldrich) from day 0 to day 5 , and two oral administrations of $\mathrm{M} 2 / 29 \mathrm{Str}^{\mathrm{R}}$ E. coli $\left(10^{9} \mathrm{CFU}\right)$ dissolved in phosphate-buffered saline were performed at days 3 and 7 of the experiment for all rats. This previously described streptomycin-pretreatment animal model [23] opens intestinal niche for $E$. coli transient colonization (4-6 weeks) by disrupting colonization resistance while preserving dense and diverse anaerobic community present in conventional animals as closely as possible.

In these experiments, treatment with RGHP and RG is shown in Figure $1 A$ \& $B$ :

- RGHP 770 mg per rat twice daily orally with supplemental $\mathrm{Na}_{2} \mathrm{CO}_{3}(5 \%)$ for 4 days (containing approximately $500 \mathrm{mg} /$ day/rat of $\mathrm{RG})$;

- RG 250 mg per rat twice daily orally with supplemental $\mathrm{Na}_{2} \mathrm{CO}_{3}(5 \%)$;

- Oral vehicle twice daily with supplemental $\mathrm{Na}_{2} \mathrm{CO}_{3}(5 \%)$.

\section{- Bacterial analyses}

Bacterial analyses were performed in samples recovered from feces on days 7 and 11 as scheduled in the experimental procedures. Fecal samples were collected throughout the course of the experiment. E. coli and other Enterobacteriaceae such as Klebsiella spp., Enterobacter spp., Serratia spp. and Citrobacter spp. (named collectively KESC), Enterococcus spp. and Proteeae spp. were monitored by using selective chromogenic agar plates (ChromID CPS, bioMérieux, Marcyl'Etoile, France). The M2/29Str ${ }^{\mathrm{R}}$ E. coli strain was analyzed by using ChromID E. coli agar plates (bioMérieux) supplemented with streptomycin sulfate $(100 \mu \mathrm{g} / \mathrm{ml})$. After $24-48 \mathrm{~h}$ incubation at $37^{\circ} \mathrm{C}$, colonies were counted. The LOD was $2 \log _{10} \mathrm{CFU} / \mathrm{g}$.

\section{- Statistical analysis}

Bacterial counts were $\log _{10}$-transformed; data were expressed as medians and interquartile ranges $(25-75 \%$ percentiles) and individual data points were displayed on graphics for each experiment. Significance for effective pairing (before and after treatment) was first verified by calculating the non parametric Spearman correlation coefficient, $r_{s}$, before testing statistical significance of differences linked to treatment by performing a Wilcoxon matched-paired signed rank test. (Prism 6, Graph Pad Software). Comparisons between vehicle-streptomycintreated rats and RGHP-streptomycin-treated or RG-streptomycin-treated rats were performed using nonparametric one-way analysis of variance (Kruskal-Wallis test) with Dunn's multiple comparison post-test (Prism 6, Graph Pad Software). Two-tail analyses were used throughout and p-values of $<0.05$ were considered statistically significant.

\section{Results}

The impact of RGHP treatment on fecal bacterial species commonly associated with UTIs was analyzed in conventional Wistar rats and in rats highly colonized with a human gut commensal $\mathrm{M} 2 / 29 \mathrm{Str}^{\mathrm{R}}$ E. coli strain sharing some common genetic traits of archetypal UPEC strains (Figure 1). Comparison with RG alone (at a dosage equivalent to that found in RGHP) was also performed in these two animal experiments (Figure 1).

\section{- Impact of RGHP on commensal E. coli populations in conventional rats}

The overall median count for rat fecal E. coli populations before treatment was 3.87 (3.65-4.48) $\log _{10}$ CFU/g feces. Oral administration of vehicle did not impact initial median fecal load ( $p=0.16,3.91$ [3.04-4.95] after vs 3.68 [2.98-4.1] before $\log _{10}$ CFU/g feces), whereas RGHP significantly reduced the initial median fecal count of E. coli $(\mathrm{p}=0.031,2.45$ [1.5-4.58] after vs 4.36 [3.55-4.74] before $\log _{10}$ $\mathrm{CFU} / \mathrm{g}$ feces, Figure 2). E. coli was no longer detectable in the feces of two of six rats treated 


\section{(A) Conventional wistar rats (gut microbiota not altered by streptomycin)}

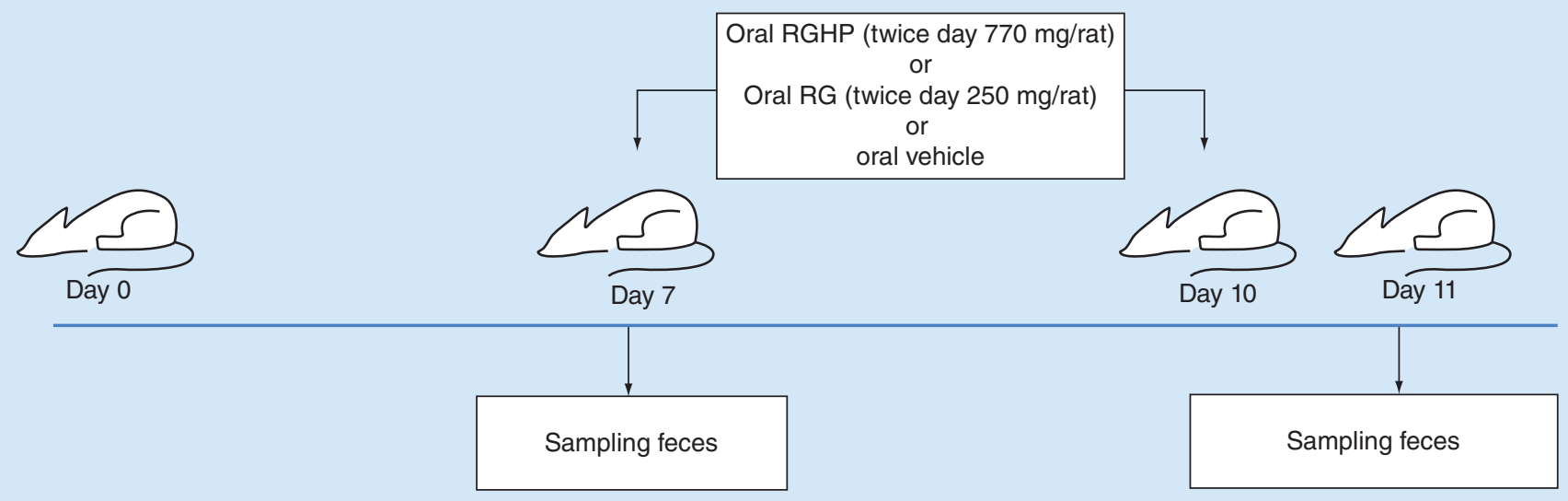

(B) Wistar rats highly colonized with streptomycin-resistant M2/29 E. coli strain

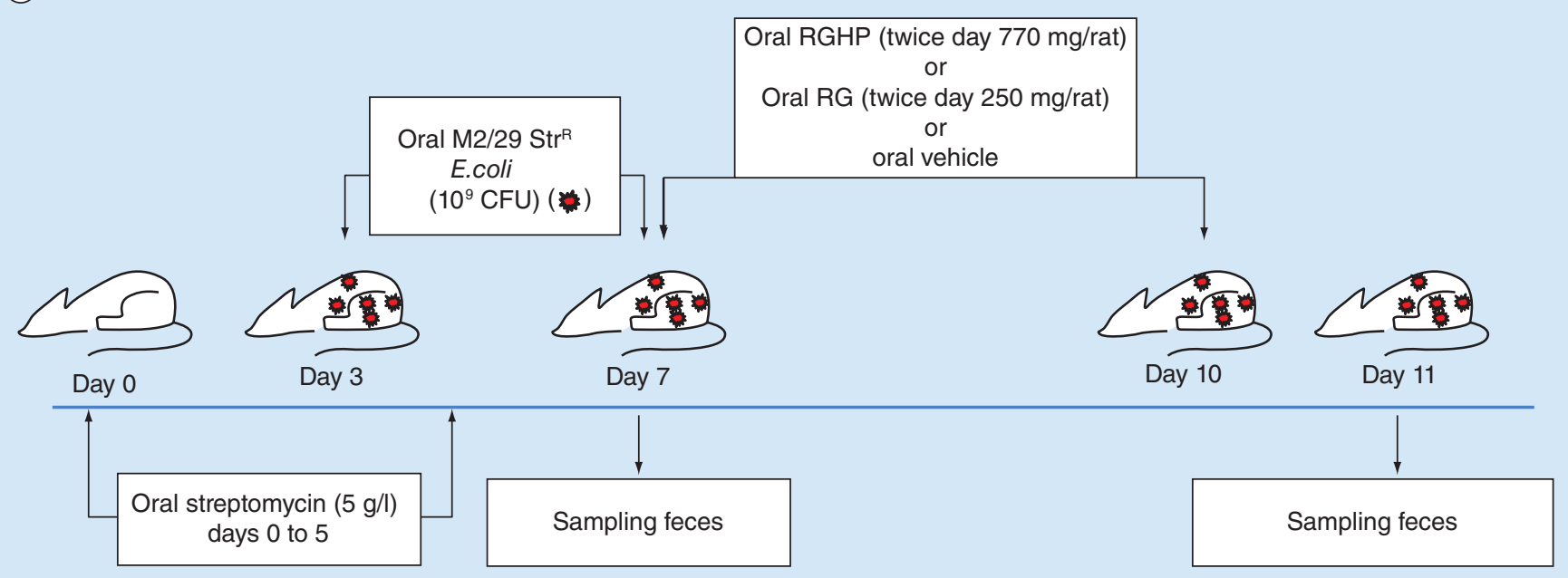

Figure 1. Experimental design. The impact of RGHP treatment and RG on selected fecal commensals was investigated on two groups of female Wistar rats. (A) Conventional Wistar rats with gut microbiota not altered by streptomycin ( $\mathrm{n}=6 \mathrm{rats} / \mathrm{group}$, vehiclecontrol, RGHP-treated and RG-treated groups). (B) Streptomycin-treated Wistar rats highly colonized with a streptomycin-resistant Escherichia coli strain (M2/29Str ${ }^{R}$ strain, $n=10$ rats/group, before treatment, vehicle-control, RGHP-treated and RG-treated groups). Oral treatments: RGHP $(2 \times 770 \mathrm{mg} /$ day/rat $), \mathrm{RG}(2 \times 250 \mathrm{mg} /$ day $)$ and vehicle $\left(2 \times\right.$ water supplemented with $\left.\mathrm{Na}_{2} \mathrm{CO}_{3}\right)$ and streptomycin sulfate $(5 \mathrm{~g} / \mathrm{l})$. Bacterial monitoring of feces (LOD $\left.=2 \log _{10} \mathrm{CFU} / \mathrm{g}\right)$.

RG: Reticulated gelatin; RGHP: Reticulated gelatin hibiscus and propolis.

with RGHP. Administration of an equivalent dose of RG alone resulted in a similar decrease of the fecal $E$. coli count as compared with initial levels before treatment $(\mathrm{p}=0.031,2.49$ [1.5-3.8] after vs 3.89 [3.56-4.49] before $\log _{10}$ $\mathrm{CFU} / \mathrm{g}$ feces, Figure 2). Rat fecal E. coli populations were no longer detectable in the feces of three of six rats treated with RG. This suggests that the RG component is the main contributor to the antagonistic activity of RGHP.
- Impact of RGHP in conventional Wistar rats on other commensal species commonly associated with UTIs

The fecal samples collected before and after treatment with RGHP, RG or vehicle were also used to estimate levels of fecal Enterococcus spp. (Figure 3A) and KESC (Figure 3B). Proteeae spp. (Proteus spp., Providencia spp. and Morganella spp.) were not detected in Wistar rat feces.

Compared with initial counts of rat commensal bacteria before treatment, RGHP-treated 
animals showed reduced fecal median counts of Enterococci ( $\mathrm{p}=0.03,3.76[3.37-4.42]$ after vs 5.39 [4.67-6.07] before $\log _{10} \mathrm{CFU} / \mathrm{g}$ feces), whereas in the vehicle group a nonsignificant increase in the median Enterococci count $(\mathrm{p}=0.16,5.81[4.73-7.19]$ after vs 4.9 [4.73-5.32] before $\log _{10} \mathrm{CFU} / \mathrm{g}$ feces) was observed in half of rats (Figure 3A). A similar but smaller median decrease in fecal Enteroccoci levels was observed in the RG treatment group as compared with initial level $(\mathrm{p}=0.03,4.4$ [3.81-4.95] after vs 5.62 [5.18-5.9] before $\log _{10}$ $\mathrm{CFU} / \mathrm{g}$ feces, Figure 3A).

Contrary to the antagonistic activities observed against E. coli and Enterococcus spp., RGHP and RG did not significantly alter the levels of colonization with KESC (Figure 3B).

\section{- Impact of RGHP on fecal E. coli in streptomycin-pretreated rats highly colonized with the M2/29Str ${ }^{\mathrm{R}}$ E. coli strain}

Using this model, the median overall initial $\mathrm{M} 2 / 29 \mathrm{Str}^{\mathrm{R}} E$. coli fecal count before treatment was 7.18 [6.93-7.37] $\log _{10}$ CFU/g feces. A decrease in the fecal $E$. coli level was observed in the vehicle group (6.45 [6.01-6.8] after vs 7.11 [6.95-7.45] before $\log _{10}$ CFU/g feces; $n$ $=8$ pairs due to the absence of data for two rats, Figure 4). This decrease due to the use of the streptomycin-pretreatment transient colonization model was not considered significant as compared with the paired initial levels $(\mathrm{p}=0.11$, Figure 4$)$. This colonization process thus remained sufficiently robust to evaluate changes during 4 days of treatment with RGHP.

Compared with the initial levels of M2/29Str ${ }^{R}$ E. coli strain before treatment, RGHP significantly reduced median fecal counts $(\mathrm{p}=0.002$, 4.94 [4.41-5.72] after vs 7.15 [6.66-7.49] before $\log _{10}$ CFU/g feces; Figure 4). Compared to vehicle-treated rats, RGHP treatment reduced the fecal counts of $E$. coli ( $\mathrm{p}=0.034,4.94$ [4.41-5.72] in RGHP-treated rats as compared with 6.45 [6.01-6.8] in vehicle-treated rats; Figure 4). A similar but less pronounced decrease in initial fecal $E$. coli counts was observed for rats treated with RG alone ( $\mathrm{p}=0.002,5.81$ [5.29-6.18] after treatment vs 7.2 [7.05-7.3] before $\log _{10} \mathrm{CFU} / \mathrm{g}$ feces; Figure 4).

\section{Discussion}

In this study, we evaluated the ability of orally administered RGHP to target and impact intestinal commensal bacteria in female Wistar rats. The focus of our study involved the most frequently isolated bacteria implicated in UTIs, in

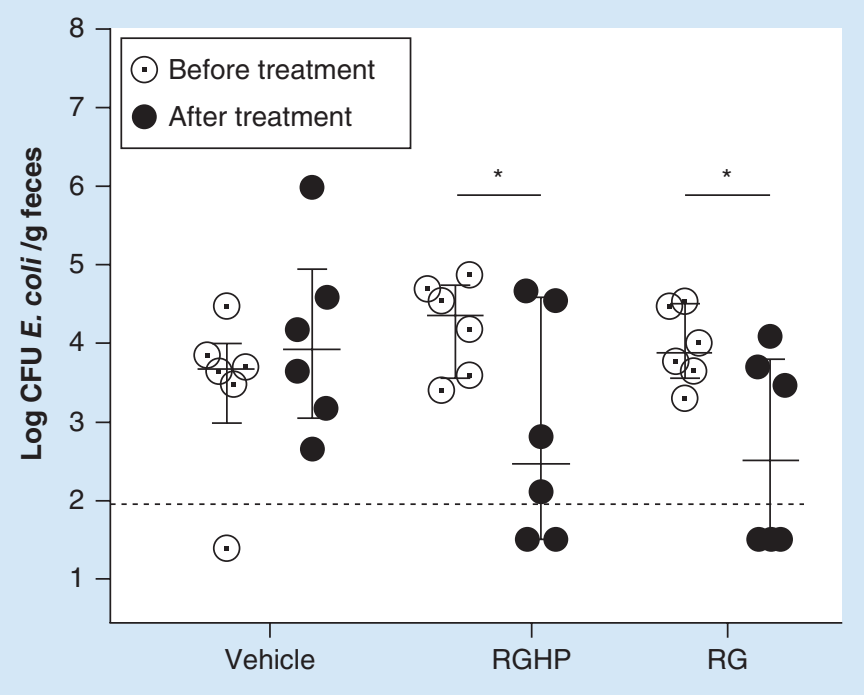

Figure 2. Impact of reticulated gelatin hibiscus and propolis and reticulated gelatin on fecal commensal Escherichia coli population of conventional female Wistar rats ( $n=6$ per group). Medians with interquartile ranges are represented and data are representative of one experiment. Levels of endogenous $E$. coli ( $\log _{10}$ CFU/g feces) were compared before and after treatments. ${ }^{*} p<0.05$; the Wilcoxon matched-pairs signed rank test. LOD $=2 \log _{10}$ CFU/g (dotted line). RG: Reticulated gelatin; RGHP: Reticulated gelatin hibiscus and propolis. 
(A)

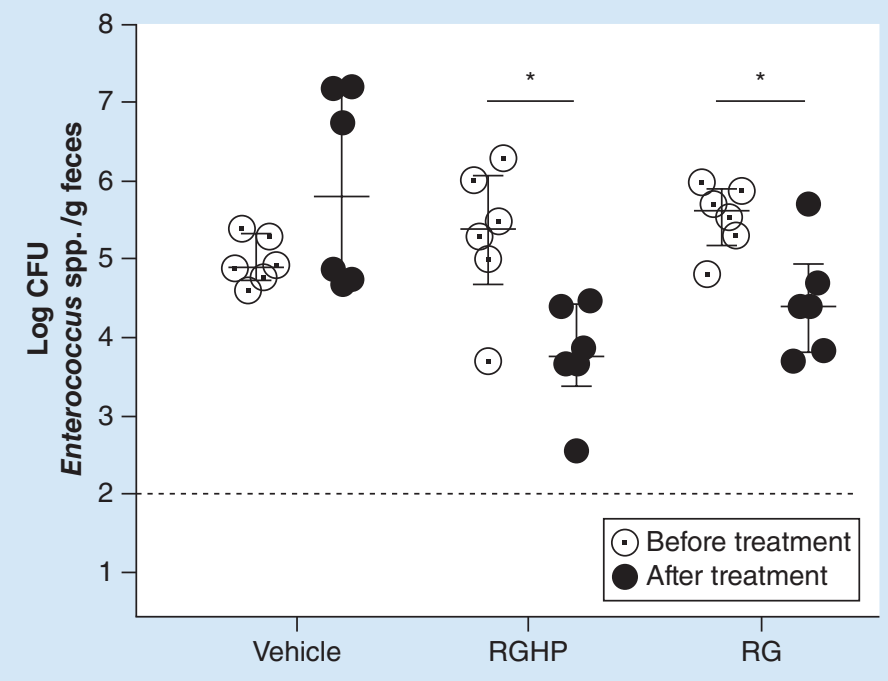

(B)

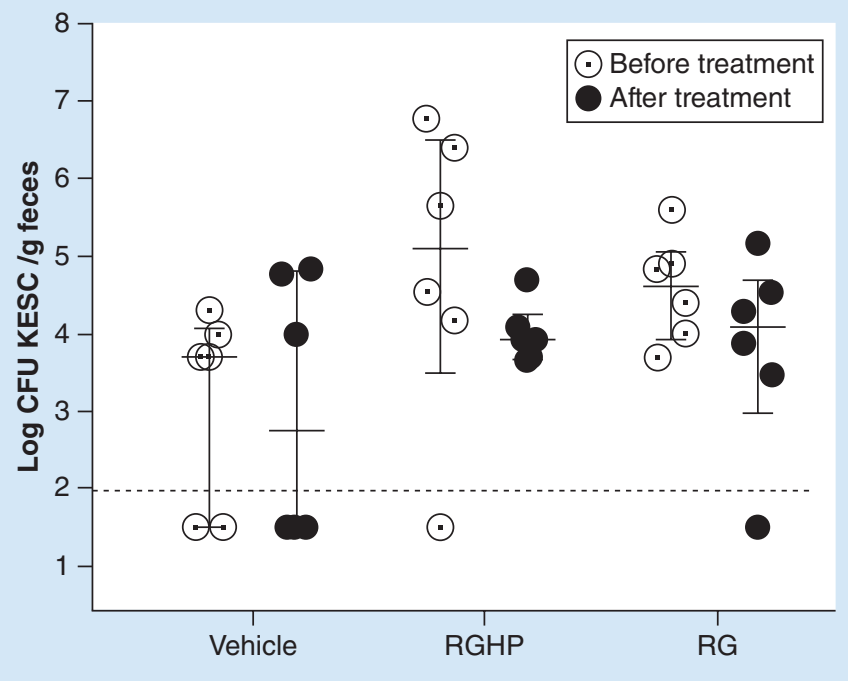

Figure 3. Impact of reticulated gelatin hibiscus and propolis and reticulated gelatin on other bacterial species commonly associated with urinary tract infections in feces of conventional female Wistar rats ( $\mathbf{n}=\mathbf{6}$ per group). Medians with interquartile ranges are represented, and data are representative of one experiment. LOD $=2 \log _{10}$ CFU/g (dotted line). (A) Levels of Enterococcus spp. ( $\log _{10}$ CFU/g feces) were compared before and after treatments; ${ }^{*} \mathrm{p}<0.05$, the Wilcoxon matched-pairs signed rank test. (B) Levels of KESC ( $\log _{10}$ CFU/g feces) were compared before and after treatments.

KESC: Klebsiella spp., Enterobacter spp., Serratia spp. and Citrobacter spp; RG: Reticulated gelatin; RGHP: Reticulated gelatin hibiscus and propolis.

other words, E. coli, Enterococcus ssp., Proteeae spp. and other Enterobacteriaceae such as KESC. The primary niche of these commensal microorganisms is the lower intestinal tract of mammals. Some species appear to be well equipped, in terms of their metabolic characteristics and fitness, to colonize extra-intestinal niches such as the urinary tract [25].

The results of the study showed that RGHP selectively reduced the fecal level of some commensal bacteria found in the conventional rat intestinal microbiota. Fecal colonization by E. coli and Enterococcus spp. was reduced following RGHP treatment and eradicated in some animals, whereas colonization by other Enterobacteriaceae (e.g., KESC) remained relatively unaffected. Unexpected presumptive Staphylococci colonies were detected in some fecal samples, but the low numbers involved and the variable response to treatment preclude any assessment of RGHP efficacy for this genus. No presumptive Proteeae spp. colonies were detected in Wistar rat feces in this study.

Mechanisms underlying the genus-dependent protective activity displayed by RGHP treatment have not been further explored in this study, and that issue still needs to be addressed. Considering that RG compound found in RGHP may preserve the appearance and elasticity of the intestinal mucus layer of colitic mice [19], we can presume that this compound may affect more specifically microbial communities occupying ecological niches such as mucus layer. Spatial distribution of bacteria such as E. coli, Enterococci from other KESC within the gut is not known and it would be of interest to localize their respective transverse niches. More specifically, the persistence of these bacteria within the outer mucus layer after RGHP treatment still needs to be evaluated. Indeed, in the case of E. coli, Li et al. recently showed that despite the fact that this facultative anaerobe has a restricted glycoside hydrolase repertoire, thus limiting its mucus metabolism, it developed adaptive strategies for population persistence specifically in the mucus as compared with another mucolytic symbiont Bacteroides thetaiotaomicron [26]. By increasing the expression of the ferric iron uptake pathway in colonic mucus, as compared with intestinal luminal uptake, E. coli improved its capability to proliferate more quickly in the outer layer of mucus (estimated half-life of about $3 \mathrm{~h}$ ) than in 


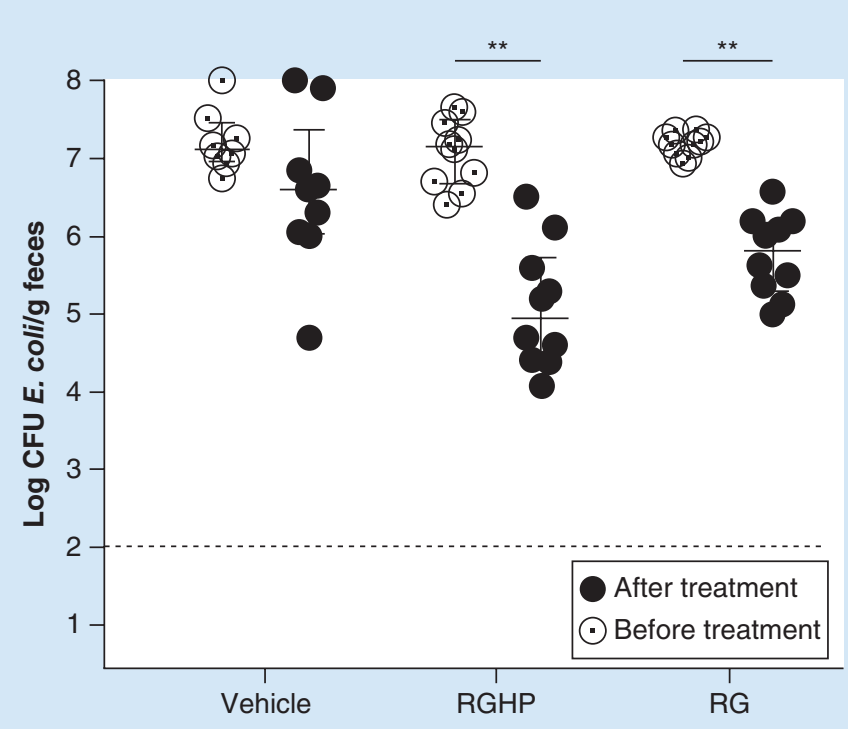

Figure 4. Impact of reticulated gelatin hibiscus and propolis and reticulated gelatin on fecal $\mathrm{M} 2 / 29 \mathrm{Str}^{\mathrm{R}}$ Escherichia coli strain orally administered in female streptomycin-treated Wistar rats ( $n=10$ per group). Medians with interquartile ranges are represented, and data are representative of one experiment. Levels of $M 2 / 29 \mathrm{Str}^{\mathrm{R}} \mathrm{E}$. coli strain ( $\log _{10} \mathrm{CFU} / \mathrm{g}$ feces) were compared before and after treatments.

${ }^{* *} \mathrm{p}<0.01$, the Wilcoxon matched-pairs signed rank test. LOD $2 \log _{10}$ CFU/g (dotted line). RG: Reticulated gelatin; RGHP: Reticulated gelatin hibiscus and propolis.

the lumen (estimated half-life of about 8 h) [26].

Following manipulation of the intestinal microbiota to encourage colonization with the $\mathrm{M} 2 / 29 \operatorname{Str}^{\mathrm{R}} E$. coli strain from the phylogenetic group B2, we showed that RGHP still retained antagonistic activity against this opportunistic uropathogenic strain. This animal model has the advantage that streptomycin pretreatment promotes both the pauciclonality and clonal dominance in feces of the orally inoculated strain of phylogroup B2, in other words, some criteria considered as favoring an enabling gut environment for increased risk of UTI [7,11].

Treatment with RG alone led to a similar spectrum of activity against both rat gut commensals and an exogenous human clone of $E$. coli strain sharing some common characteristics of the archetypal UPEC strains. Taken together, these data strongly support the concept that the $\mathrm{RG}$ component is the main contributor to the antagonistic activity of RGHP.

RG has been shown to exert protective effects against dextran sodium sulfate induced mucosal damage and, interestingly, confocal microscopy showed that the polymeric protective layer that RG forms on ulcerated mucosa limits the contact or invasion of bacteria into the intestinal epithelial layer. Taking into account the ability of $E$. coli to proliferate more quickly in the outer layer of mucus as mentioned above, we hypothesize that the protective effect of RGHP may be attributed to the formation of polymeric protective layer leading to an overall decrease in E. coli load.

\section{Conclusion}

This in vivo study clearly demonstrated that RGHP reduced fecal colonization by gut bacterial commensals such as E. coli and Enterococci or by a dominant human $E$. coli strain able to colonize extra-intestinal niches such as the urinary tract. Collectively, these results provide important new data confirming the preventive potential of RGHP to reduce the risk of contamination of the urinary tract by opportunistic bacteria of fecal origin.

\section{Future perspective}

The ascendant pathway for UTIs is well established and eradicating the uropathogen in the infected urinary tract is the primary focus of antibacterial-based therapy. Due to increased resistance in the community and in hospitals, antibiotics must be used with caution. 
Consequently, alternative methodologies for reducing the intestinal bacterial burden with a device such as RGHP is an interesting approach for the future control of UTIs and their recurrences. Indeed, if such devices can help lower the demand for antibiotics it will be an important step forward.

\section{Acknowledgements}

This article is dedicated to the memory of L Bueno, the researcher in Neuro-Gastroenterology and Nutrition team, Institute National de la Recherche Agronomique, Toulouse, France.

\section{Financial \& competing interests disclosure}

The study was sponsored by Novintethical Pharma SAGL,

Lugano, Switzerland. The authors have no other relevant affliations or financial involvement with any organization or entity with a financial interest in or financial conflict with the subject matter or materials discussed in the manuscript apart from those disclosed.

The authors thank C Gil for her assistance in writing the manuscript and Content Ed Net (Spain) for editorial assistance.

\section{Ethical conduct of research}

The authors state that they have obtained appropriate institutional review board approval or have followed the principles outlined in the Declaration of Helsinki for all human or animal experimental investigations. In addition, for investigations involving human subjects, informed consent has been obtained from the participants involved.

\section{EXECUTIVE SUMMARY}

- Forty to sixty percent of adult women experience at least one urinary tract infection (UTI) episode during their lifetime. In a global manner, UTIs represent every year more than 10 million cases in Western Europe.

- Several recurrent UTIs case reports illustrate that the intestinal microbiota is the most common immediate source of uropathogens. The uropathogenic E. coli (UPEC) strain responsible for the UTI is also present in the intestinal microbiota of $78 \%$ of women.

- Herein, we evaluated the ability of an oral class III medical device i.e., reticulated gelatin hibiscus and propolis (RGHP) recently approved in the European Union to control and prevent UTIs to limit the source of fecal contamination leading to UTIs in rat, by reducing the intestinal burden of intestinal commensals commonly involved in UTIs.

- RGHP significantly reduced fecal levels of commensal E. coli and Enterococcus spp. levels without affecting other targeted Enterobacteriaceae. Interestingly, RGPH also reduced the fecal level of a human commensal E. coli strain with uropathogenic potential.

- In conclusion, in order to limit antibacterial treatments largely used against UTIs with the associated risk of bacterial antibiotic resistance development, RGPH represents a therapeutic option through the reduction of intestinal colonization by opportunistic uropathogens.

\section{References}

Papers of special note have been highlighted as:

- of interest; $\bullet$ of considerable interest

1 Griebling TL. Urologic diseases in America project: trends in resource use for urinary tract infections in women. J. Urol. 173, 1281-1287 (2005).

2 Johansen TEB, Cek M, Naber KG, Stratchounski L, Svendsen MV, Tenke P. Hospital acquired urinary tract infections in urology departments: pathogens, susceptibility and use of antibiotics. Data from the PEP and PEAP-studies. Int. J. Antimicrob. Agents 28(Suppl. 1), S91-S107 (2006).

3 Flores-Mireles AL, Walker JN, Caparon M, Hultgren SJ. Urinary tract infections: epidemiology, mechanisms of infection and treatment options. Nat. Rev. Microbiol. 13, 269-284 (2015).

- Gives a complete overview on urinary tract infections, uropathogens, current therapeutic options, emerging problems with multidrug-resistant uropathogens and promising alternative treatments.

4 Russo TA, Stapleton A, Wenderoth S, Hooton TM, Stamm WE. Chromosomal restriction fragment length polymorphism analysis of Escherichia coli strains causing recurrent urinary tract infections in young women. J. Infect. Dis. 172, 440-445 (1995).

5 Yamamoto S, Tsukamoto T, Terai A, Kurazono H, Takeda Y, Yoshida O. Genetic evidence supporting the fecal-perinealurethral hypothesis in cystitis caused by Escherichia coli. J. Urol. 157, 1127-1129 (1997).
-• Confirms that Escherichia coli strains residing in the rectal microbiota serve as a reservoir for urinary tract infections by analyzing $\mathbf{2 7 0 0}$ clones from 9 female subjects with acute cystitis and 30 healthy women.

6 Xie J, Foxman B, Zhang L, Marrs CF. Molecular epidemiologic identification of Escherichia coli genes that are potentially involved in movement of the organism from the intestinal tract to the vagina and bladder. J. Clin. Microbiol. 44, 2434-2441 (2006).

7 Moreno E, Andreu A, Pigrau C, Kuskowski MA, Johnson JR, Prats G. Relationship between Escherichia coli strains causing acute cystitis in women and the fecal E. coli population of the host. J. Clin. Microbiol. 46, 2529-2534 (2008). 
- Authors of this paper assessed the structure of the fecal $E$. coli population in 42 women with acute cystitis and reveal that pauciclonality, fecal clonal dominance, virulence and group B2 -status are closely interwined and may characterize the commensal clones that have uropathogenic potential.

8 Herzer PJ, Inouye S, Inouye M, Whittam TS. Phylogenetic distribution of branched RNA-linked multicopy single-stranded DNA among natural isolates of Escherichia coli. J. Bacteriol. 172, 6175-6181 (1990).

9 Escobar-Paramo P, Grenet K, Le Menac'h A et al. Large-scale population structure of human commensal Escherichia coli isolates. Appl. Environ. Microbiol. 70, 5698-5700 (2004).

10 Tenaillon O, Skurnik D, Picard B, Denamur E. The population genetics of commensal Escherichia coli. Nat. Rev. Microbiol. 8, 207-217 (2010)

11 Picard B, Garcia JS, Gouriou S et al. The link between phylogeny and virulence in Escherichia coli extraintestinal infection. Infect. Immun. 67, 546-553 (1999).

12 Zhang L, Foxman B, Marrs C. Both urinary and rectal Escherichia coli isolates are dominated by strains of phylogenetic group B2. J. Clin. Microbiol. 40, 3951-3955 (2002).

13 Johnson JR, Stell AL. Extended virulence genotypes of Escherichia coli strains from patients with urosepsis in relation to phylogeny and host compromise. J. Infect. Dis. 181, 261-272 (2000).

14 Fisher K, Phillips C. The ecology, epidemiology and virulence of Enterococcus. Microbiology 155, 1749-1757 (2009).
15 García-Larrosa A, Alexe O. Efficacy and safety of a medical device versus placebo in the early treatment of patients with symptoms of urinary tract infection: a randomized controlled trial. Clin. Microbiol. 5, 233 (2016).

16 Esteban Carretero J, Durbán Reguera F, López-Argüeta Alvarez S, López Montes J. A comparative analysis of response to ORS (oral rehydration solution) vs ORS +RP in pediatric patients with acute diarrhea. Rev. Esp. Enferm. Dig. 101, 41-48 (2009).

17 Allegrini A, Costantini MA. RP for the treatment of acute diarrhoea in adults. J. Gastointest. Dig. Syst. 2, 110-115 (2012).

18 Frasca G, Cardile V, Puglia C, Bonina C, Bonina F. RP reduces the proinflammatory effects of lipopolysaccharide in human intestinal epithelial cells. Clin. Exp. Gastroenterol. 5, 61-67 (2012).

19 Scaldaferri F, Lopetuso LR, Petito V et al. Gelatin tannate ameliorates acute colitis in mice by reinforcing mucus layer and modulating gut microbiota composition: emerging role for 'gut barrier protectors' in IBD? United European Gastroenterol. J. 2, 113-122 (2014).

20 de Servi B, Moreira da Silva R, Meloni M et al. New insights into the mechanism of action of gelatine tannate for acute diarrhoea. Part 2: antibacterial activity. Presented at (oral communication): The 33rd Congress from the Groupe Francophone d'HépatoGastroentérologie et Nutrition Pédiatriques, Nantes, France, 30 March 2012.

21 Oteo J, Pérez-Vázquez M, Campos J. Extended-spectrum [beta]-lactamase producing Escherichia coli: changing epidemiology and clinical impact. Curr. Opin. Infect. Dis. 23, 320-326 (2010).

22 Pitout JD, Gregson DB, Church DL, Elsayed $S$, Laupland KB. Community-wide outbreaks of clonally related CTX-M-14 beta-lactamaseproducing Escherichia coli strains in the Calgary health region. J. Clin. Microbiol. 43, 2844-2849 (2005).

23 Myhal ML, Laux DC, Cohen PS. Relative colonizing abilities of human fecal and K 12 strains of Escherichia coli in the large intestines of streptomycin-treated mice. Eur. J. Clin. Microbiol. 1, 186-192 (1982).

24 National Research Council (US) Committee for the Update of the Guide for the Care and Use of Laboratory Animals. Guide for the Care and Use of Laboratory Animals 8th edition. doi:10.17226/12910 National Academies Press, Washington DC (2011).

25 Alteri CJ, Mobley HLT. Metabolism and fitness of urinary tract pathogens. Microbiol. Spectr. 3(3), doi:10.1128/microbiolspec. MBP-0016-2015 (2015).

26 Li H, Limenitakis JP, Fuhrer T et al. The outer mucus layer hosts a distinct intestinal microbial niche. Nat. Commun. 6, 8292 (2015).

- Highlights distinct strategies employed by some intestinal microbial communities such as $E$. coli to colonize host niches and how adaptation to stratification between the outer layer of mucus and the lumen may determine survival or extinction of bacteria in the host. 